\title{
Technical note: Development of a noninvasive respiration rate sensor for cattle
}

\author{
S. Strutzke, ${ }^{1}$ D. Fiske, ${ }^{1}$ G. Hoffmann, ${ }^{1 *}$ C. Ammon, ${ }^{1}$ W. Heuwieser,${ }^{2}$ and T. Amon ${ }^{1,3}$ \\ ${ }^{1}$ Leibniz Institute for Agricultural Engineering and Bioeconomy (ATB), Department of Engineering for Livestock Management, Max-Eyth-Allee 100, \\ 14469 Potsdam, Germany \\ ${ }^{2}$ Clinic of Animal Reproduction, and \\ ${ }^{3}$ Institute of Animal Hygiene and Environmental Health, Department of Veterinary Medicine, Freie Universität Berlin, 14163 Berlin, Germany
}

\section{ABSTRACT}

The measurement of the respiration rate $(\mathrm{RR})$ in cattle is a valuable tool for monitoring health status. Thus, an RR sensor can be essential for stress detection, especially heat stress. Heat stress leads to a deviation of the normal $R R$ and results in a decrease of milk production and fertility. Therefore, continuous monitoring of the RR can help early detection of heat stress and, thus, initiate timely counteractive actions to minimize physical stress. The most common method to measure the RR in cattle is to count the flank movement visually; however, this method is time-consuming and labor-intensive. In addition, the continuous measurement of the RR is difficult to implement and can be physically strenuous. Therefore, a device based on a differential pressure sensor that can record $\mathrm{RR}$ automatically has been developed to make continuous long-term studies possible. The aim of this study was to validate the data measured by the device with the help of a reference method. The reference method used was counting the flank movements of a total of 6 cows (Holstein-Friesian). The rear flank movements of each cow were recorded by a camera and counted independently of the device by an observer. Eight videos of 1 min each were recorded per cow. The data analysis was done with cows in 3 different body positions: dozing, lying, and standing. A total of $48 \mathrm{RR}$ measurements of the device were compared with the counted RR frequencies of the video recording. The results were highly correlated during dozing [correlation coefficient $(\mathrm{r})=$ $0.92, \mathrm{n}=13]$, lying $(\mathrm{r}=0.98, \mathrm{n}=15)$, and standing $(\mathrm{r}=0.99, \mathrm{n}=20)$. The evaluation showed that the device is suitable for automated RR counting. Further development of a marketable device is planned.

Received May 2, 2018.

Accepted September 19, 2018.

*Corresponding author: ghoffmann@atb-potsdam.de
Key words: sensor, respiration rate, stress monitoring, validation

\section{Technical Note}

The measurement of respiration rate (RR) in cattle is a diagnostic method used in practice and research. The RR is influenced by excitement, forced activity, pregnancy, high milk yield, and pathological conditions (Knickel et al., 2000). Furthermore, an increase in RR is an important indicator of stress (Gaughan et al., 2000), especially in heat-stressed animals (Hahn et al., 1997; Polsky and von Keyserlingk, 2017). Heat stress results in a decline in milk production, animal welfare, and fertility (De Rensis et al., 2015; Polsky and von Keyserlingk, 2017). If changes in RR can be detected early, targeted measures can be taken to alleviate the strain on the animal and thus prevent performance losses and increase the animal welfare.

The most common method to measure the RR in cattle is to count the flank movements visually (Milan et al., 2016); however, this method is time-consuming and labor-intensive. The continuous measurement of $\mathrm{RR}$ in long-term studies is difficult to implement, can be physically strenuous, and inevitably results in miscounts. Furthermore, the permanent presence of a person can cause stress and affect the RR of the animal, which can confound the measurements. Nonspecific flank movements, which are not caused by respiration, can also result in misinterpretation (Eigenberg et al., 2000).

Methods have been developed to measure the RR automatically. Milan et al. (2016) developed a device that monitors changes in temperature near the nostrils with a thermistor. Its advantage is the continuous recording of RR, but this method does not provide information about the strength of exhaled and inhaled pressure, which in turn can provide important information about breathing patterns (e.g., shallow as well as deep breathing), which can give information about stressful situations. Another disadvantage of thermistors is that 

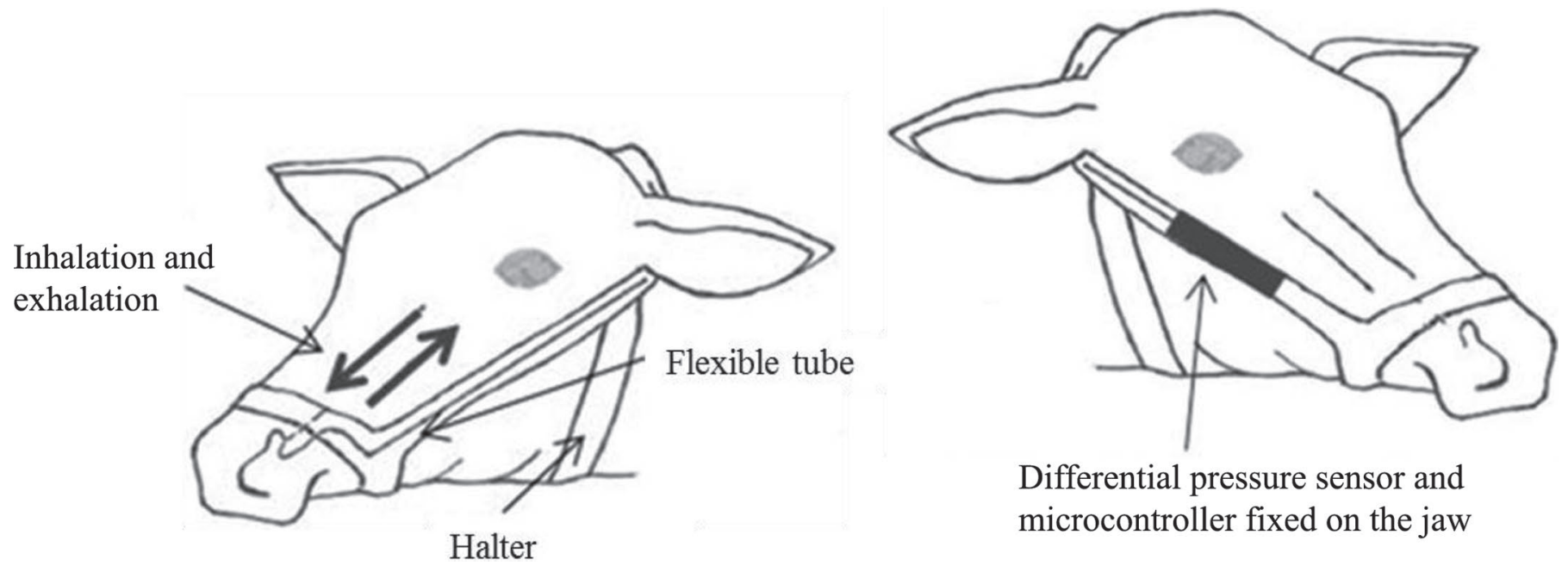

Figure 1. Construction and position of the respiration rate sensor.

their function is based upon a temperature difference between ambient and body temperature. The signal can diminish if both temperature values are close to each other. Eigenberg et al. (2000) tested a sensor originally designed for human application (Respiratory Effort Transducer, BIOPAC Systems Inc., Goleta, CA). Their device consists of a belt, which is attached around the chest of animals and measures thoracic and abdominal movements. The drawback of this method is the frequent slipping of the belt. The animal or other animals may try to move the belt. This can result in signal interference or sensor damage (Milan et al., 2016). In addition, Pastell et al. (2007) developed a system to measure $\mathrm{RR}$ via a laser distance sensor during milking. The sensor points at the side of the abdominis lateralis region of the cow and measured the movement in this region; however, the device is not mobile and continuous measurement is impossible. In addition, stress during milking may influence the RR.

The objective of our study was to develop a device and mounting hardware for RR measurement in cattle and to examine whether the new method can replace the current standard of visual counting.

For the present study, we developed a sensor device to measure $\mathrm{RR}$ that can record the output data from a continuous and automatic sensor, which is especially useful for long-term studies. The system consists of a differential pressure sensor, a microcontroller, and software to analyze the data. A halter positioned the sensor on the jaw, fixed on the right side of the head. A flexible silicon tube, running over the neck of the animal, connected one port of the pressure sensor with the nostril on the opposite side and extended $10 \mathrm{~cm}$ into the nasal cavity (Figure 1,2). The other port of the sensor was left open and hence exposed to ambient pressure.
Through a flexible silicon tube, the nasal exhalation pressure was transmitted to the pressure sensor, and the microcontroller converted the incoming analog signal from the pressure sensor to a digital signal (Figure $3)$. The pressure differential increases with flow velocity (Scott, 2008); thus, the incoming pressure (Pa) at the sensor increases at the beginning of the exhalation, which is characterized by an increase in the breathing curve (sampling rate $100 \mathrm{~Hz}$ ). At the beginning of the inhalation, the pressure at the sensor decreased and a negative pressure was generated, which resulted in a drop of the breathing curve (Figure 4). The data transmission to a server occurred via a wireless local area network (WLAN). The RR monitor included a sensor, microcontroller, and silicon tube, with a total weight of $45 \mathrm{~g}$, plus a power bank (capacity 2,600 mAh) weighing $60 \mathrm{~g}$. The dimensions of the sensor with the microcontroller were $46 \times 15 \times 25 \mathrm{~mm}$ (length $\times$ width $\times$ height).

Our experimental study using animals was approved by the State Office for Occupational Safety, Consumer Protection and Health (LAVG, Brandenburg, Germany) under the study number 2340-1-2018. The study was conducted over $2 \mathrm{~d}$ at the Educational and Experimental Center for Animal Breeding and Husbandry (LVAT, Groß Kreutz, Germany) during January 2018. Six Holstein Friesians were used in our study and data acquisition was recorded at different times. On the first day, data were taken during the day and night (1 cow 0800 to $0800 \mathrm{~h}$ the following day and 2 cows 0800 to $1800 \mathrm{~h}$; average ambient temperature $=1^{\circ} \mathrm{C}$; average relative humidity $=75.5 \%$ ) and on the second day during the day ( 3 cows 0800 to $1800 \mathrm{~h}$; average ambient temperature $=9.5^{\circ} \mathrm{C}$; average relative humidity $=$ $87.5 \%$ ). The dairy cows were housed in a freestall barn 


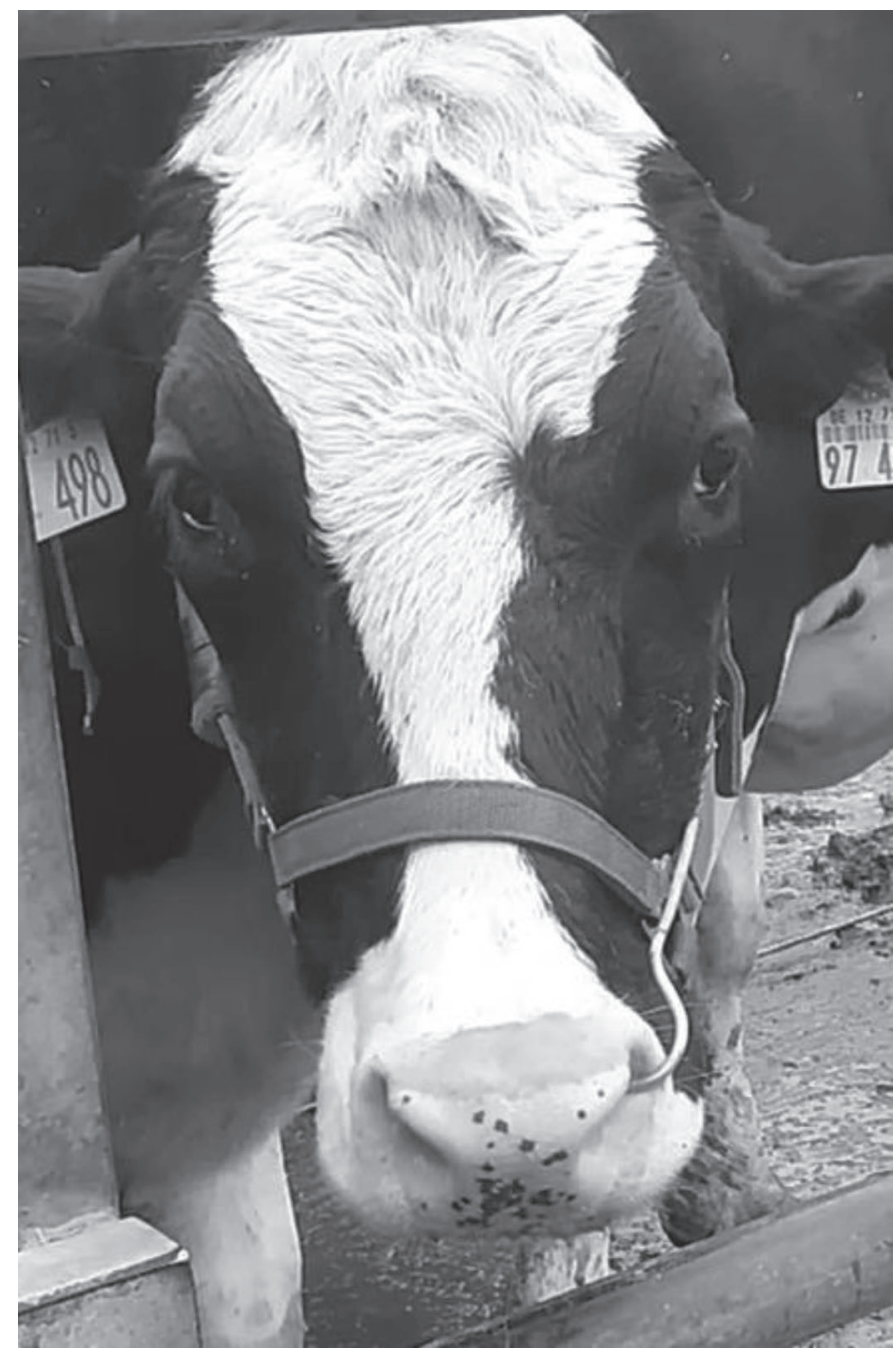

Figure 2. Cow with sensor device attached.

equipped with 53 lying cubicles (straw-lime mixture) and were part of an existing herd of 55 cows on the first day and 54 cows on the second day. The animals differed in their stage of lactation, 3 in their second lactation and 1 cow each in their first, fourth, and fifth lactation. This sample ensured that the sensor device worked at different ages and lactation stages. The animals were able to move freely in the barn during the experimental study so as not to restrict their natural behavior. Water and a TMR were freely available.

The continuous data acquisition for each test animal took $10 \mathrm{~h}$, with the exception of 1 animal, where a 24-h measurement was carried out. The 24-h measurement was done to test the robustness of the sensor device and the acceptance of it by the animal over such a long time. The RR data were logged continuously over the investigation period. Only short interruptions $(<1 \mathrm{~min})$ occurred due to replacements of the power banks. To ensure a constant recording, every power bank was exchanged after 6 h. For each examination day, 3 different cows were used for data acquisition. At the beginning, the test animals were stationed at the feeding fence and equipped with the sensor. After a short acclimatization and observation time of $30 \mathrm{~min}$, the data were recorded. In preliminary tests, animal monitoring was carried out over a period of $4 \mathrm{~h}$. During this observation, no restrictions on the behavior of the animals were obvious. All test animals had a high level of acceptance to the sensor device and a multiday acclimatization was not necessary. As a reference method for data acquisition, video recordings (Samsung Galaxy Note 10.1, Seoul, South Korea) were made of the rear flank movement while dozing (in lying cubicle, lying position, dozy with eyes half-closed, without rumination), lying (in lying cubicle, cow actively observing the environment, with and without rumination), and standing (in the walkway or cubicle, with or without rumination). It was a prerequisite for the video recording that the cow stands or lies calmly without external disturbances (e.g., other cows). In total, 8 video recordings of $5 \mathrm{~min}$ each were taken to ensure to get at least 1 min without disturbances; videos were then stored for each individual. For each body position, at least 2 data streams with a total duration of $1 \mathrm{~min}$ each were used and included in the evaluation. A light-emitting diode (LED) fixed to the sensor signaled the beginning of each video recording and was turned on manually. The LED signal was used as a marker to assign the recordings. In addition, the time of the recordings was synchronized with the time of data acquisition. The generated data were saved and graphically displayed in Excel 2010 (Microsoft Corp., Redmond, WA). The breathing pattern was counted visually (peak counting method) for a fixed period of 1 min each. An increase and subsequent decrease of the curve corresponded to 1 breath (Figure 4). Independent of the sensor data, 1 person counted the RR visually by use of the videos. For this purpose, according to the time stamp of the sensor data, the RR was counted from the video recordings for $1 \mathrm{~min}$. The flashing LED indicated the beginning and end of counting. One breath was defined as the right- or left-sided lifting and compression of the abdomen. The number of abdominal movements per minute corresponded to the determined RR.

The statistical evaluation was performed by JMP (12.0.1, SAS Institute Inc., Cary, NC) with a significance level of $\alpha=0.05$. To assess the strength of the statistical relationship between the $\mathrm{RR}$ generated by the sensor data and the visual observation data, a Bravais-Pearson correlation analysis was conducted. To describe the agreement between the visual observation and the sensor data, a matched pairs analysis was per- 


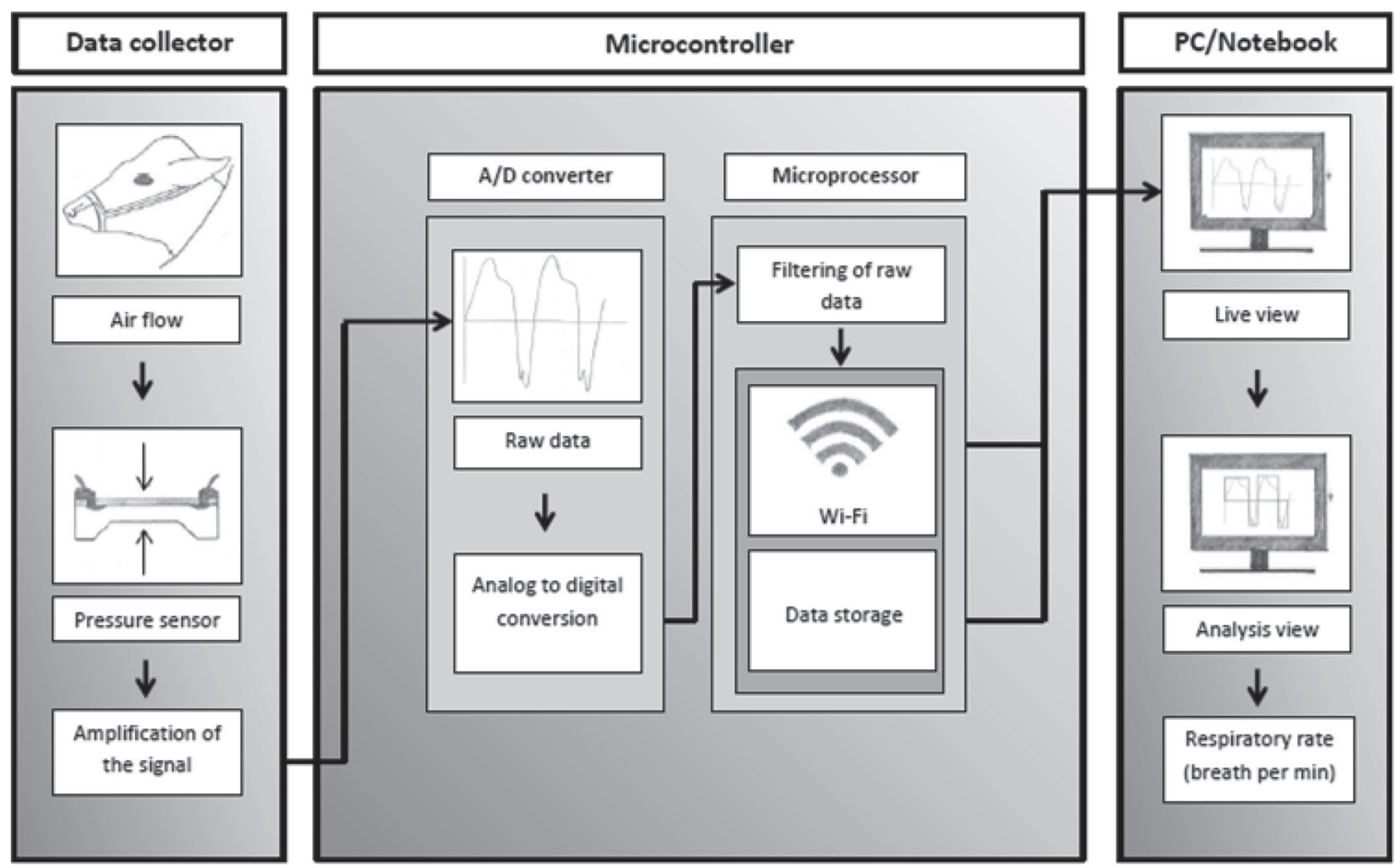

Figure 3. Flowchart of the data transmission. The generated exhalation pressure was sent to the differential pressure sensor, where it was converted into a digital signal. To reduce the noise in the raw data, the data were filtered in the microprocessor and sent to a server for evaluation. $\mathrm{A} / \mathrm{D}=$ analog to digital.

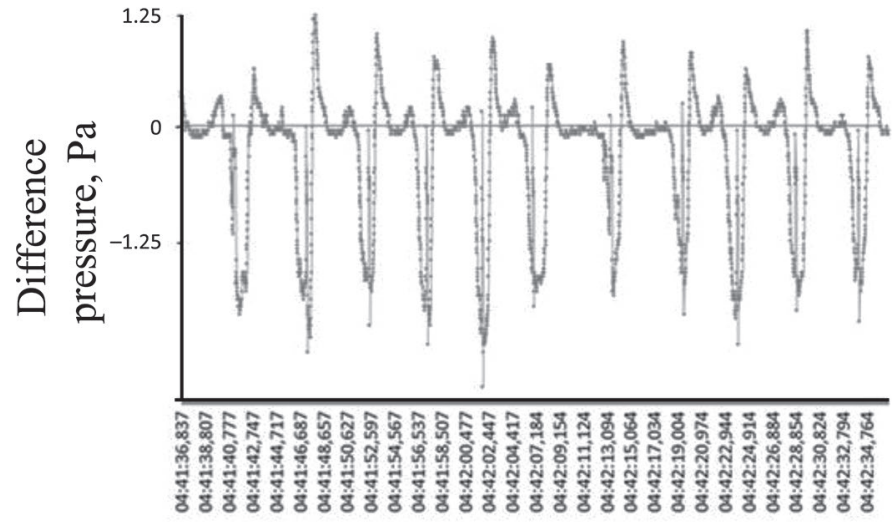

Time, hh:mm:ss,mss

Figure 4. Pressure difference measured by the sensor. The pressure triggered by the exhalation on the sensor leads to an increase in the data curve. The negative pressure triggered by the inhalation leads to a drop in the curve. formed, which includes a Tukey mean-difference plot and the results of a paired $t$-test.

The results are presented as mean-difference plots, also known as Bland-Altman plots (Bland and Altman, 1999). They show the difference between the 2 paired measurements (y-axis), and the $\mathrm{x}$-axis represents the average of the sensor data and the visual counting data (Figure 5); the horizontal line illustrates the mean difference, with the $95 \%$ confidence interval above (dotted line). Figure 4 shows converted data of the sensor signal and depicts the RR of a cow. Two peaks are present in the ascending and descending region. The pressure triggered by the exhalation over the sensor leads to an increase in the curve, visible between the minimum and the next maximum peak. The negative pressure triggered by the inhalation leads to a drop in the curve, visible between the maximum and the next minimum peak. The average pressure measurement in our study was between -5 to $5 \mathrm{~Pa}$. The zero line represents an area without breathing. The graphical representation of the $\mathrm{RR}$ demonstrates that the sensor device is able to 

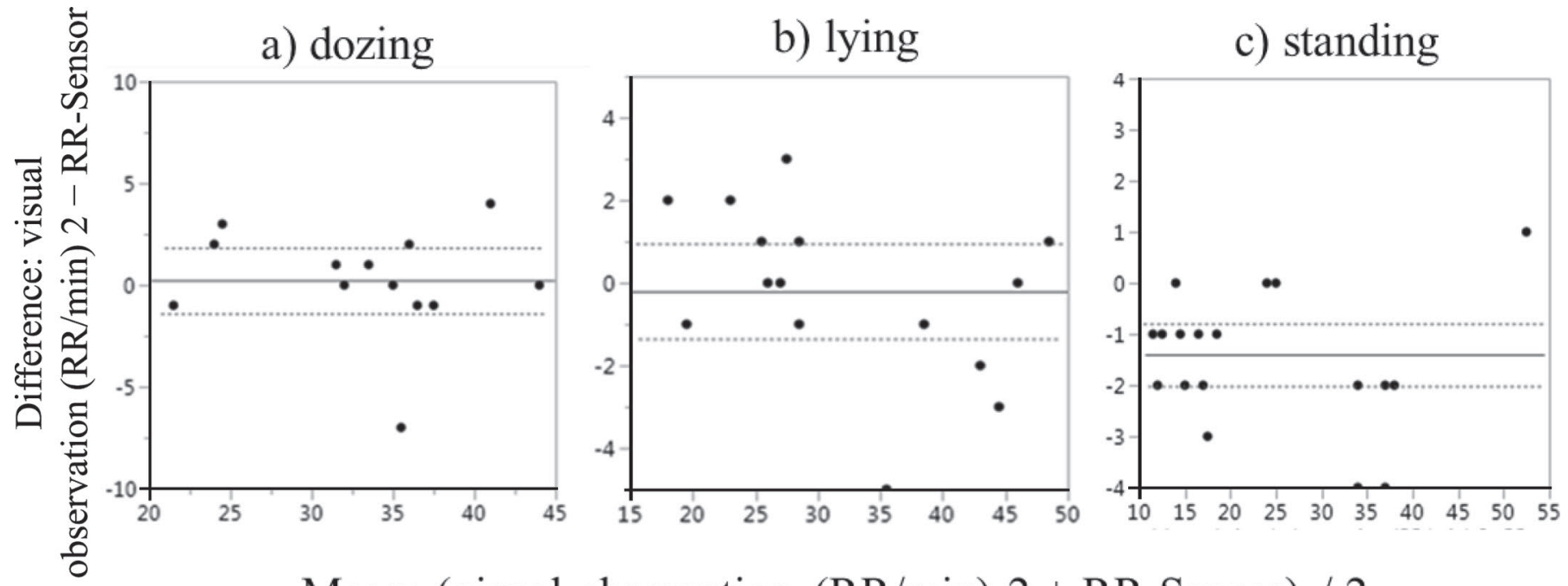

\section{Mean: (visual observation (RR/min) $2+$ RR-Sensor) / 2}

Figure 5. Difference between respiration rate (RR) during dozing (a), lying (b), and standing (c) counted by the sensor and visual observation versus mean of both methods. Data represents 1 -min recordings ( $95 \%$ CI of a, b, and c: -1.39 to $1.86,-1.35$ to 0.95 , and -2.01 to -0.78 , respectively; mean difference of $\mathrm{a}, \mathrm{b}$, and $\mathrm{c:} 0.23,-0.2$, and -1.4 , respectively).

detect RR. Over the trial period, the animals showed a high level of acceptance to the sensor device. We found no obvious outward impairment in behavior and health. Nasal discharge did not affect the recording of the RR. The sensor recorded the data of the RR constantly over the entire period with the exception of the $24-\mathrm{h}$ measurement, as the test animal lost the sensor after $22 \mathrm{~h}$. A previous test of the power bank showed that a constant $\mathrm{RR}$ recording over $8 \mathrm{~h}$ was possible. Therefore, every power bank was exchanged after $6 \mathrm{~h}$ during the trial to ensure a constant recording. The test animals had an average RR of 29 breaths per minute during the trial period with a standard deviation of 10 breaths/ min.

The results of the correlation analysis showed a high correlation coefficient and coefficient of determination for the $\mathrm{RR}$ during dozing $\left(\mathrm{r}=0.92, \mathrm{R}^{2}=0.85, \mathrm{n}=\right.$ 13) and lying $\left(r=0.98, R^{2}=0.96, n=15\right)$, as well as a homogeneous and normal distribution of differences during dozing and lying in the mean-difference plot (Figure 5a, b). The mean difference of 0.2 during dozing showed that the sensor counted 0.2 fewer breaths per minute than visual counting. The $95 \%$ confidence interval (CI) for the point estimates of the mean difference was -1.39 and 1.86 breaths $(P=0.76)$. The mean difference of -0.2 during lying showed that the sensor counted 0.2 more breaths per minute than visual counting. The CI of the mean difference was -1.35 and 0.95 breaths $(P=0.71)$. The results for dozing and lying showed no significant difference between the automated and visual counting methods.
The correlation analysis for RR during standing showed a high correlation coefficient and a high coefficient of determination $\left(\mathrm{r}=0.99, \mathrm{R}^{2}=0.99, \mathrm{n}=20\right)$. The differences plotted were not homogeneous and normally distributed (Figure 5c); the differences represent a strong one-sided shift. The mean difference of -1.4 during standing showed that the sensor counted 1.4 breaths more per minute than the visual counting. The CI of the mean difference was -2.01 and -0.79 breaths $(P<0.0001)$.

The results during standing showed a significant difference between automatic and visual counting. However, in this context it is important to clarify that the one-sided shift of the differences (when compared with 0) should be considered. This is probably due to shallow breathing or difficult countable breaths due to short-term limb movement during standing, which may have resulted in a partial counting of the RR. With repeated analysis of the video recordings or direct observations beside the animals, these missing breaths could be identified. In accordance, Milan et al. (2016), who also used the nasal cavity to measure RR, showed both fewer breaths $(\mathrm{n}=4)$ and more breaths $(\mathrm{n}=1)$ counted by the sensor (thermistor) in comparison to the visual counting, whereby no differentiation between different body positions was done. It should also be considered that counting inhalations and exhalations may be a more precise approach compared with counting flank movements, which can be triggered by other causes. Thus, the sensor value appears to be the more reliable measure compared with the visual method due 
to the position of the sensor in the nasal cavity, where the pressure sensor really measures the inhalations and exhalations of the air into or out of the lung. Misinterpretation in counting RR (e.g., triggered by movement or very shallow breathing) can be avoided and error rates (e.g., due to untrained observers; Milan et al., 2016) reduced. This is an advantage in contrast to previous studies, where the abdominal movement was measured via chest belt (Eigenberg et al., 2000) or laser (Pastell et al., 2007) to record the RR. Nevertheless, the present study must be considered as a pilot study ( $\mathrm{n}=6 \mathrm{cows}$ ), and further studies with more sensor devices based on a wider sample size are necessary to confirm our results. The RR sensor also had limitations; namely, the battery life and the mounting of the sensor must be improved in the future to prevent losses of the device. The programming of special software to automatically count RR per minute is also of importance. In addition, our method is registered for patent approval. Further research is warranted to study the relationship between stress load and the $R R$ or rather the breath depth. Studies involving heat stress are planned, as already mentioned by Hahn et al. (1997) and Polsky and von Keyserlingk (2017), because RR increased in heat-stressed animals, but to date no RR thresholds are known.

In conclusion, the results of our study showed that measuring RR by a differential pressure sensor delivers reliable data. A continuous measurement of $\mathrm{RR}$ is possible and can reduce observation time. Overall, we found that the behavior of the animals during the study was not disturbed by the flexible tube, nor was their health impaired. Abnormal behavior, such as a vehement defensive reactions or restlessness, could not be detected. Further tests and developments are planned with the software and the sensor device.

\section{ACKNOWLEDGMENTS}

We thank the staff of the Educational and Experimental Center for Animal Breeding and Husbandry (LVAT,
Groß Kreutz, Germany) for providing test animals and for their help during the study. We also acknowledge Severino Pinto, Theresa Siemens, Barbara Amon, and Susanne Demba (Leibniz Institute for Agricultural Engineering and Bioeconomy, ATB Potsdam, Germany) for their dedication in the experimental study as well as for their helpful tips and suggestions during the written preparation of the Technical Note.

\section{REFERENCES}

Bland, J. M., and D. G. Altman. 1999. Measuring agreement in method comparison studies. Stat. Methods Med. Res. 8:135-160. https: //doi.org/10.1177/096228029900800204

De Rensis, F., I. Garcia-Ispierto, and F. López-Gatius. 2015. Seasonal heat stress: Clinical implications and hormone treatments for the fertility of dairy cows. Theriogenology 84:659-666. https://doi .org/10.1016/j.theriogenology.2015.04.021.

Eigenberg, R. A., G. L. Hahn, J. A. Nienaber, T. M. Brown-Brandl, and D. E. Spiers. 2000. Development of a new respiration rate monitor for cattle. Trans. ASAE 43:723-728.

Gaughan, J. B., S. M. Holt, G. L. Hahn, T. L. Mader, and R. Eigenberg. 2000. Respiration rate - Is it a good measure of heat stress in cattle? Asian-australas. J. Anim. Sci. 13(Suppl. C):329-332.

Hahn, G. L., A. M. Parkhurst, and J. B. Gaughan. 1997. Cattle respiration rate as a function of ambient temperature. ASAE Paper NMC97. Am. Soc. Agric. Eng., St. Joseph, MI.

Knickel, U. R., Ch. Wilczek, and K. Jöst. 2000. MemoVet. Practical Guide Veterinary Medicine (Praxis-Leitfaden Tiermedizin). 3rd ed. Schattauer Publishing Company, Stuttgart, Germany.

Milan, H. F. M., A. S. C. Maia, and K. G. Gebremedhin. 2016. Technical note: Device for measuring respiration rate of cattle under field conditions. J. Anim. Sci. 94:5434-5438. https://doi.org/10.2527/ jas.2016-0904.

Pastell, M., J. Kaihilahti, A.-M. Aisla, M. Hautala, V. Poikalainen, and J. Ahokas. 2007. A system for contact-free measurement of respiration rate of dairy cows. Pages 105-109 in Precision Livestock Farming '07, Skiathos, Greece. S. Cox, ed. Wageningen Academic Publisher, Wageningen, the Netherlands.

Polsky, L., and M. A. G. von Keyserlingk. 2017. Invited review: Effects of heat stress in dairy cattle welfare. J. Dairy Sci. 100:8645-8657. https://doi.org/10.3168/jds.2017-12651.

Scott, D. M. 2008. Differential pressure sensors. Pages 94-96 in Industrial Process Sensors. CRC Press Taylor \& Francis Group, Boca Raton, FL. 